\title{
DE LA EXCLUSIÓN A LA INCLUSIÓN ACADÉMICA. EL ROL DEL MAESTRO COMO EXPLORADOR Y GENERADOR DE SITUACIONES CARGADAS DE SIGNIFICADOS ATRACTIVOS EN LA ENSEÑANZA DE LA ESTADÍSTICA
}

\author{
(Of exclusion to inclusion academic. The role of the teacher as \\ generator explorer and conditions of meanings charged attractions in \\ the teaching of statistics)
}

Susan Leasle Botero Ortiz' sbotero@uao.edu.co

Jesús Gabalán Coello² jgabalan@uao.edu.co

Generar situaciones atractivas para el aprendizaje, lograr conectar a los estudiantes, llevarlos al límite de su capacidad, potenciar su nivel, etc., son situaciones por las que han atravesado todos los que han sido profesores, en cualquier nivel de escolaridad. Esta serie de reflexiones se van haciendo más frecuentes en el devenir académico de los estudiantes, dado que cada vez es más necesario lograr mayores niveles de abstracción en torno a las temáticas y su complejidad creciente. No tener en cuenta este tipo de consideraciones es, sin lugar a dudas, una forma de exclusión académica. En la universidad, este trabajo tiene una connotación especial, dado el estudio de las disciplinas en términos de factores que influencian los planes de estudio, así como el puente inherente a la enseñanza-aprendizaje, construido en común acuerdo entre profesores y estudiantes. Este trabajo presenta una propuesta pedagógica en métodos cuantitativos que intenta plasmar la necesidad de entender la labor profesoral como un compromiso permanente, en la cual es absolutamente necesario contar con la experticia del docente para generar una serie de situaciones que coadyuven a la construcción colectiva del conocimiento, entendiendo fundamentalmente que los estudiantes pertenecen a generaciones distintas en las que probablemente la forma de adquisición de tal conocimiento ha cambiado. El profesor, consciente de este cambio, debe desarrollar estrategias pedagógicas que privilegien lo más importante: el aprendizaje.

Palabras clave: Enseñanza, Aprendizaje, Estadística, Universidad, Pedagogía, Inclusión, Constructivismo.

Abstract

Many teachers generate attractive situations for learning in order to assist students to focus more and in order to their academic capacity. As students mature, their academic expectations include complex and abstract thinking. However, there are times this can be a form of academic exclusion. In college, academic inclusion has a special meaning, because studying the variety of disciplines in terms of factors that influence the curriculum, as well as the inherent bridge associated in teaching and the learning process, a mutual agreement between teachers and students. This paper presents a pedagogical proposal using quantitative methods which tries to capture the need to understand the professorial work of a teacher including their permanent commitment, in which the teacher's expertise is absolutely necessary to generate a number of situations that contribute to the collective construction of knowledge.Teachers should understand that current students belong to a different generation and knowledge acquisition has changed; so that it implies developing new teaching strategies that prioritize the most important aspect of "learning".

Keywords: Teaching, Learning, Statistics, University, Pedagogy, Inclusion, Constructivism.

' Estadística. Magíster en Epidemiología. Catedrática de la línea de estadística. Coordinadora del Área de Estadística. Oficina de Planeación y Desarrollo Institucional. Universidad Autónoma de Occidente. Cali - Colombia.

${ }^{2}$ Ingeniero Industrial. Magíster en Ingeniería con énfasis en Ingeniería Industrial. Candidato a Doctor en Medición y Evaluación en Educación. Catedrático en la línea de métodos cuantitativos. Coordinador Gestión de la Calidad. 


\section{INTRODUCCIÓN}

Los investigadores en el área de la Didáctica han desarrollado numerosos modelos pedagógicos que buscan cambiar, evolucionar o desarrollar modelos más complejos con respecto a la efectividad del proceso de enseñanza-aprendizaje. La mayoría de estos modelos se fundamenta en el mismo enfoque; buscan, en primera medida, descubrir las concepciones de los alumnos $y$, en este sentido, llevarlos a que estos las describan y expliquen en el desarrollo de la clase por medio de actividades de exploración y de investigación, para que pongan sus ideas a prueba presentando un punto de vista científico; en síntesis, llevar a los estudiantes a explorar esta nueva concepción con el objetivo de mejorar la comprensión de los conceptos (Driver, 1983).

La exclusión académica es un riesgo latente en todo proceso de enseñanza-aprendizaje. Dicho fenómeno tiene sus manifestaciones en tres momentos fundamentales, los cuales se vuelven definitivos a la hora de determinar la probabilidad de éxito en el desarrollo de un curso: momento de entrada, momento de proceso y momento de salida.

En el "momento de entrada", a nivel universitario, la exclusión suele manifestarse desde las mismas pruebas de ingreso a la educación superior. Es común que los estudiantes con bajos desempeños no suelan garantizar su acceso a las instituciones de educación pública. Otra posible forma de exclusión a la entrada, se manifiesta cuando en un curso los estudiantes no logran adquirir los conocimientos básicos de las asignaturas prerrequisitos que garanticen una adecuada promoción a la asignatura siguiente.

Del momento del proceso forman parte todas aquellas actividades desarrolladas en el marco de la enseñanza-aprendizaje. La ausencia de intervenciones didácticas adecuadas puede coadyuvar a que los estudiantes se desconecten de la problemática central bajo estudio. En este caso se presenta una forma de exclusión que es relativa a los medios en los cuales se lleva a cabo la práctica profesoral en función del aprendizaje de los estudiantes.
Por último, la exclusión en el "momento de salida" tiene una de sus manifestaciones en las pruebas estatales de final de carrera. Estas pruebas tienen el objetivo, entre otros, de comprobar el desarrollo de competencias de los estudiantes próximos a culminar los programas académicos de pregrado que ofrecen las instituciones de educación superior, así como servir de fuente de información para la construcción de indicadores de evaluación de la calidad de los programas e instituciones de educación superior, tanto como del servicio público educativo en general.

Dichas pruebas son entonces un elemento que sirve para seleccionar a los estudiantes en términos de las competencias adquiridas y poco a poco se convierten en criterios de juicio para la participación en becas o inserción al sector productivo.

Como se pudo observar sobre los momentos de entrada y salida, es inocuo e improcedente el trabajo didáctico. En esta dirección, el peso para las instituciones de educación superior se concentra en el proceso, para que este garantice cobertura, calidad y equidad.

Los procesos de nivelación de estudiantes con deficiencias en la entrada, así como las oportunas y efectivas estrategias pedagógicas, permiten que los estudiantes en dificultad logren ubicarse en el promedio deseable para el curso y los que cuentan con mayores desarrollos puedan potenciar sus fortalezas.

\section{Marco de REFERENCIA}

El constructivismo se concentra en la creación de herramientas cognitivas que reflejan la sabiduría, los deseos y las experiencias de los individuos; en este sentido, se vuelve totalmente innecesaria la sola adquisición de conceptos, contenidos o abstracciones sin dimensionamiento. Para que el aprendizaje sea exitoso, debería contar con tres factores fundamentales: concepto -conocimiento-, actividad-ejercitación-y cultura -contexto- (Bednar \& Cunningham, 199I). 
El modelo de cambio conceptual, desarrollado por Peter W. Hewson, demanda que el profesor involucre las concepciones de los estudiantes, creando una situación didáctica en la cual se suscita un conflicto conceptual que no podrá ver su solución sin la acomodación de sus concepciones iniciales, en términos de las nuevas concepciones presentadas por el profesor (Hewson, 198I).

En este sentido, el estudiante es un sujeto activo $y$, con sus conocimientos previos, toma posición con argumentos y va construyendo en conjunto con el profesor sus procesos de aprendizaje, llegando a desarrollos mucho más rigurosos y significativos.

El profesor, bajo este enfoque, deberá plantear situaciones con sentidos y significados, reconociendo que la construcción de nuevo conocimiento para el estudiante pasa por la reflexión a partir de sus conocimientos previos. En esta dirección, las situaciones deben aproximarse al contexto cultural en el cual se desenvuelve el estudiante para que este las encuentre atractivas y convergentes en sus problemáticas disciplinares, profesionales y personales.

Kenneth A. Strike and George J. Posner, precisaron las condiciones más favorables para el cambio conceptual. Es conveniente, en primera medida, que los estudiantes experimenten un cierto grado de insatisfacción en relación con sus concepciones de partida; además, conviene que la nueva concepción que se les presenta sea en su percepción, bastante fecunda (Strike \& Posner, 1982).

En este contexto, el rol del profesor es el de implementar los requisitos que permitan modificar el estado de las condiciones en juego; por ejemplo, mostrando que las concepciones iniciales no pueden explicar ciertos fenómenos mientras que otras sí; asegurándose que las nuevas concepciones reemplacen las anteriores.

Por supuesto, tal enfoque implica cambios importantes de los participantes en cuanto a su concepción de rol y responsabilidad, tanto de profesores como de estudiantes. Esto requiere igualmente de que un clima de confianza reine en la clase, de forma que el profesor y el estudiante no se sientan intimidados por cuestionamientos y posteriores evaluaciones.

Otros investigadores como Nadine Bednarz y Catherine Garnier (1989), y Jean-Pierre Astolfi y Brigitte Peterfalvi (1993), han retomado la noción de conflicto insistiendo sobre la importancia de suscitar un conflicto de carácter sociocognitivo sobre todos los estudiantes en el salón de clases.

Será entonces sobre estos obstáculos epistemológicos que deberá centrarse todo el trabajo didáctico. Según el modelo propuesto, debe conducirse a los estudiantes a tomar conciencia de la concepción que provoca el obstáculo, lo que suscitará un conflicto sociocognitivo, con el objetivo de "golpear" el obstáculo en cuestión, para proponer a los estudiantes un modelo explicativo-alternativo (Astolfi \& Peterfalvi, 1993).

\section{APROXIMACIÓN AL MODELO CONSTRUCTIVISTA}

La perspectiva constructivista y su integración a prácticas educativas, parece fuertemente atractiva. De tal forma, los modelos pedagógicos que adoptan tal perspectiva proponen generalmente actividades de aprendizaje que suelen motivar a los estudiantes al más alto nivel.

Esta se compone de actividades auténticas y significativas que sitúan a los estudiantes en el centro de su aprendizaje, movilizando sus conocimientos anteriores tanto en términos de saberes como de saber hacer y saber ser.

El modelo constructivista privilegia la reflexión, meta cognitiva que enfrentan los estudiantes, los inician en los conceptos relativos a la naturaleza del conocimiento científico $y$ a sus modelos de producción, permitiéndoles tomar conciencia de su propio modo de pensamiento. 


\section{LA ESTADÍSTICA COMO EJE TRANSVERSAL}

La utilización de variados recursos estilísticos, artísticos y lúdicos, orienta la relación significativa de las matemáticas con otras disciplinas y con la vida misma, y permite establecer relaciones entre temas por desarrollar y estimular el trabajo en la clase de matemáticas (Henao, 2005).

La estadística es una disciplina de amplia utilidad en todas las áreas del conocimiento; por tal motivo, su enseñanza no está dirigida exclusivamente hacia los profesionales de esta área; también lo está hacia otros profesionales y ciudadanos en general que deben analizar e interpretar la información para tomar sus propias decisiones. La toma de decisiones acertadas depende de la capacidad de análisis de estos profesionales y esta, a su vez, se relaciona con el desarrollo de un país en todos sus niveles: social, económico y político. Al jugar la Estadística un papel importante para la sociedad, como un motor de desarrollo, su enseñanza se convierte en tema de investigación.

En la actualidad existe bastante material sobre las temáticas que aborda la Estadística; sin embargo, es escaso el material o las investigaciones que orienten al profesor en las dificultades a las que se enfrenta para su enseñanza y en la solución de los problemas didácticos que la misma plantea, lo que definitivamente entorpece el proceso de lograr trasmitir los conocimientos estadísticos.

\section{LA PROPUESTA}

Al iniciar un curso de Estadística, los profesores se enfrentan a alumnos con diferentes niveles de conocimientos previos; en este sentido, David P. Ausubel y otros, mencionan entre los principios de la psicología educativa que, “(...) El factor más importante que influye en el aprendizaje es lo que el alumno ya sabe. Averígüese esto y enséñese consecuentemente" (Ausubel, Novak, Hanesian, \& al., 1983); por lo tanto, los conocimientos previos de los alumnos, usados fuera de contexto, generan respuestas incorrectas e interfieren en el momento de adquirir un nuevo conocimiento.

Otro aspecto importante al que se enfrentan los profesores, son las deficiencias de conocimientos matemáticos en sus alumnos necesarios para abordar la Estadística. En los siguientes párrafos se hará la propuesta de algunas estrategias didácticas para enfrentar las dificultades mencionadas.

\section{ABRIENDO LA CAJA NEGRA}

Con respecto a los conocimientos matemáticos y estadísticos que se necesitan en Estadística, una buena práctica que se puede desarrollar es el diagnóstico de estos conocimientos previos, pues como lo mencionan los autores mencionados anteriormente, si se conoce lo que el alumno ya sabe, se puede aprovechar esto para la enseñanza de los nuevos conceptos. Conocer los conceptos, las herramientas adquiridas y la utilización que hacen de estas los estudiantes en el momento de dar solución a una situación problemática, es una ventaja para el desarrollo que el profesor pueda dar a su curso. Este diagnóstico también puede ofrecer la otra cara de la situación: las deficiencias de algunas bases importantes para enfrentar la asignatura. El conocimiento por parte del profesor de este otro aspecto del estudiante, le dará una buena base para realizar su propuesta metodológica.

\section{UN “AVATAR” AL MUNDO REAL DE LA ESTADÍSTICA}

Según Carmen Batanero (200I) y Álvaro M. Ordoñez (2004), presentar al estudiante situaciones positivas o negativas de la vida diaria, que se relacionen directa $o$ indirectamente con el tema tratado en la asignatura, posibilitan el aprendizaje desde la aplicación. Bajo esta propuesta, el profesor puede presentar la utilidad de la Estadística en las diferentes áreas del conocimiento, presentando situaciones reales en un mundo virtual, en el cual el estudiante "ava- 
tar" es quien maneja las variables y puede ir experimentando y resolviendo dichas situaciones con los conceptos que se le han proporcionado en clase.

Aunque en ese momento no tenga el conocimiento completo de los conceptos estadísticos necesarios, el estudiante podrá hacerse consciente de la importancia que tienen estos en su futura profesión y en la resolución de problemas cotidianos.

\section{SITUACIONES INOLVIDABLES}

Entre las teorías del aprendizaje, el constructivismo sostiene que el conocimiento no es una copia de la realidad, sino una construcción constante realizada por el individuo, derivada de la interacción de dos factores: el entorno que lo rodea y sus propias habilidades. Dicha construcción la arma con los conceptos y esquemas (que ya posee), con la relación (que ya estableció entre ellos) y con una situación en particular (Carretero, 1997).

Apoyado en esta teoría, el profesor puede recurrir a situaciones con las cuales el estudiante tenga una fuerte conexión, y de las cuales ya tiene información, para construir un nuevo análisis y revisar la decisión tomada inicialmente. De este modo, el profesor revisará y hará evidente el concepto utilizado, posiblemente de forma empírica por el estudiante, y podrá ajustarlo o cambiarlo si es necesario, presentándole conceptos nuevos como otras alternativas para resolver la misma situación.

\section{TODO ES MÁS FÁCIL DESDE LA}

\section{PRÁCTICA}

Autores como Ordoñez (2004), opinan que comprender el significado y las implicaciones de los conceptos es más importante que memorizar una lista de fórmulas complejas, que pueden causar en los estudiantes angustia innecesaria, alejándolos de los aspectos realmente importantes del curso. En este sentido, al iniciar la presentación de un concepto, la enseñanza des- de la aplicación y los ejercicios prácticos facilitan la comprensión y consolidación de los conceptos.

Otro elemento que permite descubrir y entender los conceptos fundamentales en las diferentes etapas del aprendizaje es el juego. Sobre este último, Batanero (200I) recomienda que los estudiantes experimenten y construyan los conceptos mediante la manipulación de material y recursos didácticos.

\section{EJEMPLOS EXTREMOS}

Los antiejemplos suelen ser de gran importancia en la enseñanza de la Estadística. Citar ejemplos con situaciones que brinden choques a las emociones de los estudiantes puede ser de utilidad. Una situación puntual es la explicación de las desventajas de la utilización del promedio y su sensibilidad a valores extremos. Llevar a los estudiantes a la situación hipotética de una zarza ardiente en la cabeza y los pies en el hielo, implica que en términos reales estaría de por sí inerte, pero en promedio estaría bien dada la semisuma de las temperaturas.

\section{JUGANDO A SER (ROL)}

Esta estrategia permite que los estudiantes tomen un protagonismo activo a través de representar un personaje que deba resolver misterios o emprender aventuras. Estos desafíos constituyen el centro del aprendizaje, porque para poder avanzar, los estudiantes deberán hacer uso de su conocimiento estadístico.

Estos desafíos pueden darse a nivel:

- Interpretativo

- Argumentativo

- Propositivo

\section{CONSTRUYENDO LA HISTORIA}

En esta propuesta, el estudiante es quien construye las historias y elije los personajes y sus roles dentro de ella. Debe construir las situaciones que posteriormente serán validadas 
por el profesor, y que permitirán que el estudiante tome el papel del profesor en la construcción y validación de los supuestos de entrada en Estadística. Preparar todo el libreto de la situación suele requerir de un esfuerzo mucho mayor que ser partícipe en la historia. En este caso, deberá ser el responsable de la generación completa del escenario.

\section{GAZAPOS EN LECTURAS DE MEDIOS}

\section{MASIVOS}

Una estrategia para la familiarización con el pensamiento estadístico es la reflexión en torno a los postulados de la Estadística mal utilizados en los medios masivos de comunicación. Es importante que los estudiantes reconozcan el error como medio para la construcción del conocimiento.

En esta medida, saber lo que no es adecuado es un primer paso a nivel inductivo para reconocer lo que es adecuado. Esta aproximación es importante porque permite al estudiante saber que la estadística es una herramienta contextual y su interpretación dependerá del entorno en el cual se desarrolle.

\section{ELEMENTOS DE REFLEXIÓN}

¿Qué destrezas debe adquirir un estudiante que inicia su formación en Estadística para desarrollar habilidades en la toma de decisiones profesionales en presencia de variabilidad e incertidumbre?

Colin Mallows afirma que “(...) El pensamiento estadístico tiene que ver con la relación de datos cuantitativos con un problema del mundo real, a menudo en presencia de variabilidad e incertidumbre. Intenta hacer preciso y explícito lo que los datos dicen sobre el problema de interés" (Mallows, 1998). Bajo esta definición, se espera desarrollar en el estudiante la capacidad de abordar problemas reales con presencia de variabilidad e incertidumbre, para lo cual es primordial lograr ese razonamiento y pensamiento estadístico del que habla el autor, que inicia con la comprensión y utilización del lenguaje y las herramientas básicas de la Estadística.

También se busca que el estudiante pueda formalizar problemas en las diferentes áreas del conocimiento, modelando los fenómenos registrados en el entorno, en los que la presencia de variabilidad e incertidumbre lo enfrentará a desarrollar su capacidad argumentativa y a implementar métodos para recolectar, sistematizar y analizar los diferentes tipos de datos que validen o refuten la distribución del fenómeno estudiado. Finalmente, cuando el estudiante sepa lo que significan los términos y tenga claro los conceptos, será capaz de reconocer e interpretar plenamente los datos estadísticos relacionados con su profesión.

¿Qué habilidades debe tener un profesor que imparte la asignatura de Estadística para desarrollar las competencias del estudiante que inicia su formación?

Reconocer la diversidad de sus estudiantes y las diferentes formas de aprendizaje que pueden tener, es una actividad que plantea un reto interesante, pues se sabe que una de las grandes dificultades es la heterogeneidad de las bases matemáticas y de comprensión lectora con las que llegan los estudiantes a estos cursos. Para ello, debe pasar de la educación basada en el profesor a la educación centrada en el alumno, en la que la comunicación es fundamental para conseguir que el estudiante se sienta cómodo al presentar sus inquietudes, sin temor a la crítica o la burla. Igualmente, es necesario un alto grado de percepción del profesor en el momento de detectar dificultades de aprendizaje dentro de un grupo.

También se requiere que el profesor tenga iniciativa y creatividad para poner en marcha las diferentes actividades que soporten cada una de las estrategias mencionadas anteriormente; para ello, dentro de su rol debe implementar las condiciones que le permitan un ambiente de confianza con sus alumnos, para plantearles situaciones muy cercanas y familiares a su entorno, en las que ellos puedan reconocerse y proponer soluciones haciendo uso de nuevos conocimientos. 
Otro elemento al que debemos referirnos es el uso de las tecnologías en la investigación y la pedagogía. El profesor debe tener conocimientos y competencias sobre modelos de enseñanza y modelos de evaluación cuando se utilizan las tecnologías de la información y la comunicación (TICs). Adquirir destreza en el manejo de las TIC requiere de buena disposición y una actitud de formación continua.

\section{CONCLUSIONES}

Se ha construido una propuesta de aproximación pedagógica que emplea diversas estrategias desde un enfoque constructivista, que fomentan el aprendizaje de la estadística y sus niveles de competencia. Como herramienta contextual, la estadística debe ser inducida en los estudiantes de una manera poco convencional, dado que al tornarse transversal será la praxis la que oriente los desafíos interpretativos en función de las áreas disciplinares que se aborden.

Por ejemplo, el significado de la desviación estándar para un licenciado en Ciencias de la Educación tendrá que ver con la homogeneidad (situación deseable) de los procesos educativos, mientras que para el ingeniero industrial tendrá sentido en temáticas específicas relacionadas con aseguramiento de calidad y muestreo estadístico de procesos. Esto implica desarrollar en los estudiantes la capacidad de contextualizar la información obtenida a través de la estadística para el servicio de sus disciplinas.

Este trabajo se circunscribe a una propuesta académica que, como tal, es susceptible de mejoramiento y se encuentra en construcción continua, marcando una línea de pensamiento pedagógico bien definida, en términos de garantizar que se propicien ambientes en los cuales el estudiante sea el centro del proceso de construcción de conocimiento. En esta dirección, la propuesta más que desarrollarse desde la perspectiva de la enseñanza, lo hace desde el aprendizaje mediado por estrategias pedagógicas.
Acorde con lo anterior, es imperativo comprender el papel del profesor como aquel individuo que hace todo lo "humanamente posible" para que las personas que tiene al frente comprendan las nociones fundamentales, conceptos y aplicaciones del objeto del aprendizaje. Esta actitud solo se logra bajo el afecto genuino, que posibilita que al profesor le importe el progreso de sus estudiantes, tanto como a un padre le interesa el éxito de sus hijos.

Por tanto, es importante la coexistencia del concepto de motivación, dado que el profesor debe ser, por constitución natural, una persona que permita a sus estudiantes darse la oportunidad de ir más allá, generando un clima de búsqueda permanente del conocimiento en cada encuentro; esto lo convierte en una persona sensible que recrea la realidad y hace de cada encuentro un espacio único e irrepetible.

Esta propuesta es una reflexión que propende por evidenciar algunas estrategias que llevan a la construcción de actividades y situaciones en las que el estudiante participe activamente, conozca de primera mano las consecuencias de su proceder disciplinar y en las que puede salir adelante en situaciones complejas de su realidad a través de la construcción de su nueva concepción.

Para ello, es menester que el profesor ponga en jaque los conceptos y paradigmas entrantes. En virtud de ello, el estudiante experimentará su obstáculo epistemológico y tendrá la oportunidad de tomar posición con respecto al conocimiento. Este momento requiere de gran destreza y compromiso del profesor, así como de la innovación y creatividad direccionadas desde el saber disciplinar. Es por este motivo que el profesor, más que un rol de enseñanza, adquiere un nuevo nombre: explorador y generador de situaciones cargadas de significados atractivos 
Referencias bibliográficas

Astolfi, J., \& Peterfalvi, B. (1993). Obstacles et construction de situations didactiques en sciences expérimentales. ASTER, I6 (I) I03-II4.

Ausubel, D. P., Novak, J., Hanesian, H., \& al. (1983). Psicología educativa: un punto de vista cognoscitivo. Mexico D.F.: Trillas.

Batanero, C. (200I). Didáctica de la Estadística. Recuperado el I5 de julio de 20I4, de Grupo de Educación Estadística Universidad de Granada: http://www.ugr.es/ batanero/pages/ARTICULOS/didacticaestadistica.pdf

Bednar, A. K., Cunningham, D., \& al. (199I). Theory into practice: How do we link? En A. G. (Ed.), Instructional Technology: Past, Present and Future. Denver, Colorado: Libraries Unlimited.

Bednarz, N., \& Garnier, C. (1989). Construction des savoirs: obstacles et conflits. Montreal: Cirade et Agences d'Arc.

Carretero, M. (1997). ¿Qué es el constructivismo?: desarrollo cognitivo del aprendizaje. Mexico D.F.: Progreso.

Driver, R. (1983). The pupil as a scientist? Milton Keynes, England: Open University Press.

Henao, R. D. (2005). Un viaje literario en la enseñanza de las matemáticas. Madellín: Nuevo Horizonte.

Hewson, P. W. (198I). A conceptual change approach to learning science. European Journal of Science Aducation, (3) 4, 383-396.

Larochelle, M., \& Bednarz, N. (1994). À propos du constructivisme et de l'éducation. Revue des sciences de l'éducation, I0(I), 5-19.

Larochelle, M., Désautels, J., \& François, R. (1992). Autour de l'idée de science: itinéraires cognitifs d'étudiants et d'étudiantes. QuébecBruxelles: Presses de l'Université Laval-De Boeck-Wesmael.

Mallows, C. (1998). The zeroth problem. American Statistician, 52, I-9.

Ordóñez, A. (2004). Didáctica de la Estadística. Quetzaltenango, Guatemala: Unidad de Investigación y Publicaciones, Universidad Rafael Landívar.

Strike, K. A., \& Posner, G. J. (1982). Conceptual change and science teaching. European Journal of Science Education, 4, 23I-240. 